\title{
Modelo Predictivo de la No Unión de Tibia
}

\section{Tibia Non Union Predictive Model} \author{
Luis Alberto Bahamonde $2,3,5,8$ (0) \\ ${ }^{1}$ Departamento de Ortopedia y Traumatología, Hospital Clínico \\ Mutual de Seguridad, Jefe de Equipo de Trauma de Extremidad \\ Inferior, Santiago, Chile \\ ${ }^{2}$ Departamento de Ortopedia y Traumatología, Hospital Clínico \\ Mutual de Seguridad, Traumatólogo de Equipo de Trauma de \\ Extremidad Inferior, Santiago, Chile \\ ${ }^{3}$ Departamento de Ortopedia y Traumatología, Hospital Clínico \\ Universidad de Chile, Equipo de Rodilla, Santiago, Chile \\ ${ }^{4}$ Hospital Clínico Mutual de Seguridad, Médico general, Santiago, Chile \\ ${ }^{5}$ Departamento de Ortopedia y Traumatología, Hospital Clínico \\ Universidad de Chile, Equipo de Tumores Óseos, Santiago, Chile \\ ${ }^{6}$ Programa de Ortopedia y Traumatología, Universidad de Valparaíso, \\ Hospital Clínico Mutual de Seguridad, Médico Residente, Santiago, Chile \\ ${ }^{7}$ Programa de Ortopedia y Traumatología, Universidad de Chile, Hospital \\ Clínico Universidad de Chile, Médico Residente, Santiago, Chile \\ ${ }^{8}$ Departamento de Ortopedia y Traumatología, Clínica Alemana de \\ Santiago, Tumores Óseos, Santiago, Chile \\ Rev Chil Ortop Traumatol 2020;61:18-22.
}

Álvaro Igor Zamorano ${ }^{1,2,3(-)}$ Carlos Felipe Albarrán ${ }^{40}$ Pierluca Zecchetto $2,3,5$ (1)

Andrés Sebastián Oyarzún ${ }^{4}$ Luis Felipe Ramirez ${ }^{4}$ Gabriel Ignacio Durán ${ }^{6}$ Matías Alejandro Vaccia7(i)

Address for correspondence Álvaro Igor Zamorano, MD,

\section{Resumen \\ Palabras Clave \\ - fractura de tibia \\ - modelo predictivo \\ - no unión de tibia \\ Objetivos Estimar un modelo predictivo para la no-unión en pacientes que presentan fractura de tibia. \\ Materiales y Métodos Estudio de cohorte retrospectivo, en pacientes con fractura de tibia operadas entre 2012 y 2018, con un mínimo de 12 meses de seguimiento, excluyendo amputaciones traumáticas. Realizamos un modelo de regresión logística con 13 variables descritas en la literatura. Se descartaron las variables estadísticamente no significativas y las que no causaban efecto de confusión. Se evaluó la bondad de ajuste mediante el test de Hosmer-Lemeshow y la discriminación del modelo con la curva ROC. \\ Resultados Se incluyeron 411 fracturas de tibia, las variables estadísticamente significa- tivas fueron: exposición ósea $\mathrm{OR}=2,57(\mathrm{IC}: 1,15-5,75, p=0,022)$, diabetes $\mathrm{OR}=3,29$ (IC:1,37-7,91, $p=0,008)$ y uso de tutor externo $O R=1,77(I C: 0,81-3,85)$, el que tuvo efecto de confusión. La bondad de ajuste demostró que los datos se ajustan adecuada- mente al modelo $(p=0,35)$. La curva ROC demuestra un $70,91 \%$ de poder discriminatorio. Al evaluar aisladamente las fracturas expuestas, no hubo asociación estadísticamente significativa con ninguna variable. \\ Discusión Al evaluar el modelo, obtuvimos una asociación estadísticamente signifi- cativa entre: no unión, exposición ósea, diabetes y uso de tutor externo, información concordante con la literatura. Al estudiar el subgrupo de fracturas expuestas, las demás variables son estadísticamente no significativas. Eso refleja que la exposición ósea es la variable que confiere mayor riesgo. El seguimiento adecuado de esos pacientes es fundamental dado este alto riesgo de evolucionar con no-unión.}

received

November 19, 2019

accepted

March 20, 2020
DOI https://doi.org/

10.1055/s-0040-1709722. ISSN $0716-4548$.
Copyright (e) 2020 by Thieme Revinter

Publicações Ltda, Rio de Janeiro, Brazil
License terms

(1) (1) $\Theta \circledast$ 


\section{Abstract \\ Keywords \\ - predictive model \\ - tibial fracture \\ - tibial non union}

Conclusión En nuestra serie, la exposición ósea es el factor de riesgo más importante para presentar no unión de tibia.

Objectives Estimate a predictive model for non-union in patients presenting with a tibial fracture.

Materials and Methods Retrospective cohort study in patients with tibia fractures operated between 2012 and 2018, with a minimum follow-up of 12 months, excluding traumatic amputations. We performed a multivariate logistic regression model with 13 variables described in the literature. The variables that were statistically non-significant and those variables that do not cause confusion, were discarded. Goodness of fit was evaluated using the Hosmer-Lemeshow test and the discrimination of the model with the ROC curve.

Results 411 tibial fractures were included, the statistically significant variables were: bone exposure $\mathrm{OR}=2.57(\mathrm{Cl}: 1.15-5.75, p=0.022)$, diabetes $\mathrm{OR}=3.29(\mathrm{Cl}: 1.37-7.91$, $p=0.008)$ and use of external fixation $\mathrm{OR}=1.77(\mathrm{Cl}: 0.81-3.85)$, being included in the model because of its confounding effect. Goodness of fit demonstrates that the data fit the model adequately $(p=0.35)$. The ROC curve demonstrates $70.91 \%$ discriminatory power. When evaluating the exposed fractures in isolation, there was no statistically significant association with any variable.

Discussion When evaluating the model, we obtained a statistically significant association between non-union, bone exposure, diabetes and use of external fixation, being consistent with the literature. When studying the subset of exposed fractures, the other variables are statistically non-significant. This reflects that bone exposure is the variable that confers the greatest risk. Proper follow-up of these patients is essential given this high risk of evolving with non-union.

Conclusion In our series, bone exposure is the most important risk factor for presenting tibial non-union

\section{Introducción}

Las fracturas de tibia tienen una incidencia de 21,5 cada 100.000 habitantes año, ${ }^{1}$ siendo la fractura más frecuente de huesos largos. Una de las complicaciones que puede tener una fractura es la no unión, que se presenta en el $5 \%-15 \%$ de los casos. ${ }^{2}$

La no unión es una patología de causa multifactorial, en las que se incluyen la edad, diabetes, tabaquismo, exposición ósea, localización de la fractura, lesiones concomitantes, ausencia de carga precoz, tipo de osteosíntesis, necesidad y tiempo de uso de tutor externo y por último pero no menos relevante, la necesidad y tiempo de espera para cobertura cutánea tipo colgajo. ${ }^{3-6}$

Hasta la fecha no se ha diseñado un modelo predictivo para la no unión de tibia que involucre los distintos factores de riesgo implicados.

El objetivo de nuestro estudio es estimar un modelo de regresión logística multivariado que permita predecir la no unión en aquellos pacientes con fractura de tibia. Nuestra hipótesis es que existe una asociación significativa entre la no unión de tibia y: exposición ósea, tabaquismo, diabetes, ausencia de carga precoz, uso de tutor externo, tipo de osteosíntesis, localización de la fractura, necesidad de colgajo y tiempo de espera para cobertura con colgajo.

\section{Materiales y Métodos}

Estudio de cohorte retrospectivo. Se evaluó un total de 504 pacientes con fractura de tibia controladas por nuestro equipo entre enero del 2012 y julio del 2018. Se incluyeron a todos aquellos pacientes que fueron operados por el equipo, con seguimiento clínico mensual y seguimiento radiológico bimensual. Se excluyeron aquellos pacientes que tuvieron amputación traumática y los que se siguieron por menos de 12 meses. El proceso de revisión de fichas y el subsecuente estudio, cuentan con la aprobación del comité de ética clínica de nuestra institución.

Designamos como variable dependiente la presencia de no unión. Las variables independientes evaluadas fueron: exposición ósea, tiempo de espera para cobertura con colgajo, edad, diabetes, tabaquismo, necesidad de uso de tutor externo, días con fijador externo, carga precoz, localización de fractura (tercio medio, proximal o distal) y tipo de osteosíntesis (clavo endomedular o placa).

Para diagnosticar no unión de tibia, nos basamos en por lo menos uno de los siguientes criterios: movilidad en el sitio de la fractura al examen físico, movilidad en el sitio de la fractura en la evaluación radioscópica, ausencia de consolidación en las 4 corticales de las proyecciones AP y L o 
scanner que demuestra ausencia de intento de consolidación en sitio de fractura. ${ }^{7}$

Para evaluar el efecto de confusión de las variables independientes, se realizó el modelo de regresión logística que incluyó a todas las variables descritas y posteriormente se excluyó todas aquellas variables con valor $P>0,25$. En aquellas variables con valores P entre 0,05 y 0,25 , se evaluó el porcentaje de cambio que causaban sobre el OR calculado de la exposición ósea. En el modelo final, se incluyeron todas aquellas variables que provocaron un cambio entre $15 \%-20 \%$ ${ }^{8}$ y las variables con un valor $p<0,05$.

Se estimó el modelo de regresión logística multivariado significativo, y posteriormente evaluamos la bondad de ajuste mediante el uso del test de Hosmer-Lemeshow (4 subgrupos) ${ }^{9}$. Finalmente se evalúo la discriminación del modelo con el uso de la curva ROC. El cálculo de las pruebas estadísticas se realizó mediante el uso del programa STATA 14.0

\section{Resultados}

Fueron incluidos 411 pacientes al aplicar los criterios de inclusión y exclusión. La caracterización de la muestra se especifica en la - Tabla 1.

Se evaluó el modelo de regresión logística global ( - Tabla 2), retirando a todas aquellas variables con valor $\mathrm{P}>0,25$ : edad, necesidad de cirugía plástica, tipo de osteosíntesis y carga precoz.

Se evaluó la presencia de confusión al realizar una regresión logística multivariada entre exposición ósea y aquellas variables con valor P entre 0,05 y 0,25 , descartando todas aquellas variables que no produjeron un cambio del OR del $>15 \%$ de la exposición ósea, ${ }^{8}$ entre las variables estaban
Tabla 2 Resultados del modelo de regresión logística global

\begin{tabular}{|l|l|l|l|l|}
\hline & OR & SE & $P$ & IC 95\% (OR) \\
\hline Exposición & 2.69 & 1.21 & 0.027 & $1.12-6.50$ \\
\hline Edad & 1.003 & 0.01 & 0.809 & $0.98-1.03$ \\
\hline Diabetes & 3.17 & 1.72 & 0.034 & $1.09-9.12$ \\
\hline Tabaquismo & 0.51 & 0.24 & 0.152 & $0.20-1.28$ \\
\hline Fijador Ext. & 2.25 & 1.11 & 0.101 & $0.85-5.90$ \\
\hline Días c/FE & 0.96 & 0.03 & 0.143 & $0.91-1.01$ \\
\hline Cirugía plástica & 1.34 & 0.70 & 0.582 & $0.48-3.74$ \\
\hline $\begin{array}{l}\text { Días de espera } \\
\text { para cobertura } \\
\text { (colgajo) }\end{array}$ & 1.02 & 0.02 & 0.209 & $0.99-1.06$ \\
\hline Carga & 0.74 & 0.28 & 0.431 & $0.35-1.57$ \\
\hline Proximal & 2.09 & 1.26 & 0.22 & $0.64-6.81$ \\
\hline Distal & 1.33 & 0.56 & 0.496 & $0.58-3.04$ \\
\hline Clavo & 0.72 & 0.33 & 0.476 & $0.30-1.76$ \\
\hline
\end{tabular}

cantidad de días con tutor externo, tabaquismo y localización de la fractura. Se mantuvo en el modelo el uso de tutor externo, siendo considerado como variable de confusión por la variación significativa que provoca sobre el OR de la exposición ósea ( - Tabla 3$)^{8} .^{8}$

El modelo estima un OR de 2,38 (IC 1,05-5,41) si la fractura es expuesta, un OR de 1,60 (IC 0,72-3,55) si hubo necesidad de usar fijador externo, OR 1,03 (IC 0,99-1,06) por cada día de espera de cobertura con colgajo y un OR de 3,34 (IC $1,37-8,16$ ) si el paciente tiene diabetes ( - Tabla 4).

Al realizar el test de Hosmer-Lemeshow, resultó que nuestros datos no se adecúan correctamente a nuestro

Tabla 1 Características demográficas de la muestra incluida

\begin{tabular}{|c|c|c|c|c|c|}
\hline Variables & $\mathbf{n}$ & Promedio & DS & Mediana & Rango \\
\hline Edad & 411 & 41,1 & 13,49 & 41 & $18-82$ \\
\hline Sexo & $\begin{array}{l}\text { Mujeres }=21 \\
\text { Hombres }=142\end{array}$ & & & & \\
\hline Diabetes & $34(8,54 \%)$ & & & & \\
\hline Obesidad & $33(8,53 \%)$ & & & & \\
\hline Tabaquismo & $86(22,93 \%)$ & & & & \\
\hline Exposición ósea & $186(42,26 \%)$ & & & & \\
\hline Fijador externo & $174(42,34 \%)$ & & & & \\
\hline Días con fijador externo & 411 & 5,26 & 12,7 & 0 & $0-150$ \\
\hline Cirugía plástica (colgajo) & $38(9,25 \%)$ & & & & \\
\hline Días de espera para cobertura (colgajo) & 411 & 1.89 & 8.19 & 0 & $0-83$ \\
\hline Carga precoz & $312(76,1 \%)$ & & & & \\
\hline Localización de la fractura & $\begin{array}{l}\text { Proximales }=23 \\
\text { Medio }=279 \\
\text { Distal }=109\end{array}$ & & & & \\
\hline OTS & $\begin{array}{l}\text { Clavo }=334(82,06 \%) \\
\text { Placa }=73(17,94 \%)\end{array}$ & & & & \\
\hline No unión & $46(11,19 \%)$ & & & & \\
\hline
\end{tabular}


Tabla 3 Resultado de las posibles variables confundentes del modelo. Se evaluaron todas aquellas variables que en el modelo de regresión logística global tuviesen un valor P 0.05-0.25

\begin{tabular}{|l|l|l|l|l|}
\hline & $\begin{array}{l}\text { OR de } \\
\text { exposición } \\
\text { ósea }\end{array}$ & $\begin{array}{l}\text { \% Cambio } \\
\text { OR de } \\
\text { exposición } \\
\text { ósea }\end{array}$ & $P$ & IC \\
\hline $\begin{array}{l}\text { Exposición } \\
\text { ósea }\end{array}$ & 3.52 & $\mp$ & $<0.001$ & $1.80-6.91$ \\
\hline Días con TE & 3.66 & $3.98 \%$ & 0.705 & $0.97-1.02$ \\
\hline $\begin{array}{l}\text { Tutor } \\
\text { externo }\end{array}$ & 2.40 & $31.82 \%$ & 0.080 & $0.92-4.31$ \\
\hline Tabaquismo & 3.66 & $3.98 \%$ & 0.586 & $0.36-1.77$ \\
\hline $\begin{array}{l}\text { Fracturas } \\
\text { proximales }\end{array}$ & 3.39 & $3.69 \%$ & 0.272 & $0.63-5.29$ \\
\hline $\begin{array}{l}\text { Fracturas } \\
\text { distales }\end{array}$ & 3.60 & $2.27 \%$ & 0.347 & $0.70-2.76$ \\
\hline
\end{tabular}

Tabla 4 OR y valores $\mathrm{P}$ de modelo de regresión logística multivariado para la predicción de la no unión de tibia. Se observa presencia de un valor $\mathrm{P}>0.05$ en la variable Días de espera para cobertura (colgajo)

\begin{tabular}{|l|l|l|l|l|}
\hline & OR & EE & $P$ & IC \\
\hline Exposición & 2.38 & 0.998 & 0.038 & $1.05-5.41$ \\
\hline Diabetes & 3.34 & 1.522 & 0.008 & $1.37-8.16$ \\
\hline Fijador externo & 1.60 & 0.650 & 0.251 & $0.72-3.55$ \\
\hline $\begin{array}{l}\text { Días de espera } \\
\text { para cobertura } \\
\text { (colgajo) }\end{array}$ & 1.03 & 0.015 & 0.064 & $0.99-1.06$ \\
\hline
\end{tabular}

modelo seleccionado ( $p=0,029$ ) (5 subgrupos), por lo que decidimos retirar la variable días de espera para cobertura con colgajo, al no ser una variable de confusión y al ser la variable con mayor valor P $(0,064$, IC $0,99-1,06)$.

Posteriormente se evaluó nuestro modelo de regresión logística final donde el modelo estima un OR de 2,57 (IC 1,15-5,75) para los pacientes con exposición ósea, OR de 3,29 (IC 1,37-7,91) para los pacientes con diabetes y un OR de 1,77 (IC $0,81-3,85$ ) para los pacientes que hubiesen pasado por tutor externo (-Tabla 5).

Tabla 5 OR y valores $\mathrm{P}$ del modelo de regresión logística multivariado final. Se descarta la variable Días de espera para cobertura (colgajo), dado que no corresponde a una variable confundente y tampoco a una variable estadísticamente significativa para poder predecir la no unión de tibi

\begin{tabular}{|l|l|l|l|l|}
\hline & OR & SE & $P$ & $I C$ \\
\hline Exposición ósea & 2.57 & 1.056 & 0.022 & $1.15-5.75$ \\
\hline Diabetes & 3.29 & 1.47 & 0.008 & $1.37-7.91$ \\
\hline Fijador externo & 1.77 & 0.702 & 0.150 & $0.81-3.85$ \\
\hline
\end{tabular}

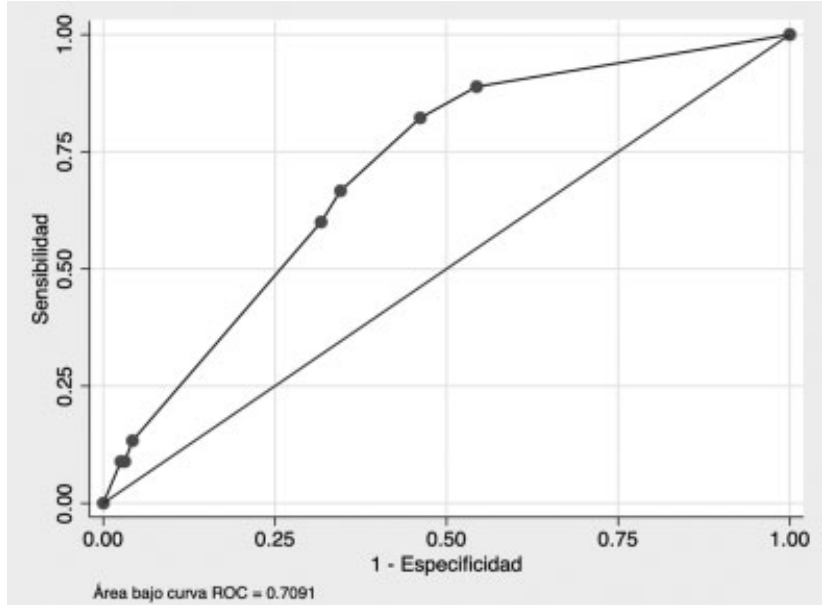

Fig. 1 Curva ROC con un área bajo la curva de 70.91\%. Existe un $70.91 \%$ de capacidad del modelo para poder predecir correctamente a los sujetos según el evento que realmente presentan.

Tabla 6 OR y valores $P$ de un modelo de regresión logística global que incluye solo a las fracturas de tibia expuestas

\begin{tabular}{|l|l|l|l|l|}
\hline & OR & EE & $P$ & IC 95\% \\
\hline Edad & 0.99 & 0.017 & 0.561 & $0.96-1.02$ \\
\hline Diabetes & 1.56 & 1.272 & 0.585 & $0.32-7.71$ \\
\hline Tabaquismo & 0.72 & 0.376 & 0.523 & $0.26-2.00$ \\
\hline Fijador Ext. & 1.68 & 0.986 & 0.380 & $0.53-5.31$ \\
\hline Días c/FE & 0.97 & 0.024 & 0.266 & $0.93-1.02$ \\
\hline Cirugía plástica & 0.99 & 0.561 & 0.992 & $0.33-3.01$ \\
\hline $\begin{array}{l}\text { Días de espera } \\
\text { para cobertura } \\
\text { (colgajo) }\end{array}$ & 1.03 & 0.020 & 0.117 & $0.99-1.07$ \\
\hline Carga & 0.90 & 0.413 & 0.816 & $0.37-2.21$ \\
\hline Proximal & 1.45 & 1.085 & 0.616 & $0.34-6.28$ \\
\hline Distal & 2.23 & 1.094 & 0.103 & $0.85-5.83$ \\
\hline Clavo & 0.65 & 0.351 & 0.238 & $0.38-2.25$ \\
\hline
\end{tabular}

Se realizó el test de Hosmer-Lemeshow y con nuestro nuevo modelo, demostramos que los datos se adecúan correctamente a nuestro modelo seleccionado ( $p=0,35$ ) (4 subgrupos).

Finalmente, calculamos la curva ROC de nuestro modelo, la cual demuestra un poder de discriminación del 70,91\% ( - Fig. 1).

Al evaluar la regresión logística multivariada usando fractura expuesta como variable dependiente, no hubo asociaciones estadísticamente significativas con las otras variables (-Tabla 6).

\section{Discusión}

Hasta la fecha no se ha intentado diseñar un modelo explicativo de la no unión de tibia. Nosotros logramos diseñar un modelo predictivo que se ajusta adecuadamente a nuestra serie de casos, destacando que la variable más importante fue la exposición ósea. 
Distintos estudios han reportado un OR de 2,56 hasta $4,00^{10,11}$ para presentar no unión de tibia, en aquellos pacientes que presentaron fractura expuesta, hallazgo consistente con lo estimado con el OR estimado por nuestro modelo 3,52 (IC 1,80-6,91).

Nuestra serie tiene una tasa baja de no unión del $11,16 \%$ en comparación a la literatura en que se describe la prevalencia de no unión hasta el $80 \%$ en algunas series de casos. ${ }^{12}$

El hallazgo más significativo de nuestro trabajo, es la asociación que existe entre la no unión de tibia y la exposición ósea. Al evaluar las fracturas de tibia expuestas por separado, no hubo asociaciones estadísticamente significativas entre las variables y la no unión. Eso refleja que la variable más importante para presentar no unión corresponde a la exposición ósea. El grado de exposición ósea depende directamente de la energía del traumatismo, en donde ocurre daño extenso de partes blandas, desperiostización y alteración en la vasculatura local. ${ }^{13}$ Cabe destacar que, pese a que no incluimos en el modelo la evaluación de infección profunda relacionada a fractura, ella es clínicamente importante ya que las fracturas expuestas de tibia tienen mayor riesgo de infección profunda relacionada a la fractura, alcanzando hasta un $4,3 \%{ }^{14}$

La limitación más importante de este estudio es el diseño que presentamos. Al ser un estudio de cohorte retrospectivo, existe el riesgo de presentar sesgo de selección por pérdida de seguimiento, si bien al ser una institución que provee un seguro médico con compensación a los pacientes, no logramos el seguimiento del $100 \%$ de los pacientes intervenidos por nuestro equipo por distintas razones, pero es muy cercano a él.

En la literatura se describe que el gasto por paciente que presenta no unión de tibia es en promedio 26.000 USD, contando la estadía hospitalaria, cirugía, osteosíntesis ocupadas, medicamentos y licencia médica, ${ }^{10}$ siendo un gasto no menor a nivel nacional si se piensa que la no unión de tibia varía entre $5 \%-15 \%{ }^{2}$

Consideramos que es de suma importancia realizar un seguimiento estricto de los pacientes con fractura expuesta, pues tenemos la ventaja de que la gran mayoría de nuestra población es cautiva, aunque lamentablemente eso no ocurre en todos los centros. Recomendamos controles clínicos mensuales y controles radiológicos bimensuales con evaluación de scanner en ausencia de progresión de la consolidación en 2 controles radiológicos seriados asociado a ausencia de mejoría sintomática. En el caso de ausencia de progresión de la consolidación con scanner, se debe plantear la opción de realizar algún procedimiento quirúrgico a determinar según las características de la no unión en cada paciente.

\section{Conclusiones}

La exposición ósea tiene una fuerte asociación con la no unión de tibia. El resto de las variables estudiadas en nuestra serie, no son estadísticamente significativas al quitar la exposición ósea de por medio. Es muy importante un seguimiento riguroso y una alta sospecha clínica en todos aquellos pacientes que sufran una fractura expuesta de tibia para tratar complicaciones de forma precoz.

Conflicto de Intereses

Los autores declaran no tener ningún conflicto de intereses.

\section{Bibliografía}

1 Court-Brown CM, Caesar B. Epidemiology of adult fractures: A review. Injury 2006;37(08):691-697

2 Mills LA, Aitken SA, Simpson AHRW. The risk of non-union per fracture: current myths and revised figures from a population of over 4 million adults. Acta Orthop 2017;88(04):434-439

3 Karladani AH, Granhed H, Kärrholm J, Styf J. The influence of fracture etiology and type on fracture healing: a review of 104 consecutive tibial shaft fractures. Arch Orthop Trauma Surg 2001; 121(06):325-328

4 Copuroglu C, Calori GM, Giannoudis PV. Fracture non-union: who is at risk? Injury 2013;44(11):1379-1382

5 Gaston MS, Simpson AH. Inhibition of fracture healing. J Bone Joint Surg Br 2007;89(12):1553-1560

6 Calori GM, Albisetti W, Agus A, Iori S, Tagliabue L. Risk factors contributing to fracture non-unions. Injury 2007;38(Suppl 2): S11-S18

7 Brinker MR, O'Connor DP, Monla YT, Earthman TP, Earthman TP. Metabolic and endocrine abnormalities in patients with nonunions. J Orthop Trauma 2007;21(08):557-570

8 de Irala J, Martínez-González MA, Guillén Grima F. Qué es una variable de confusión? Med Clin (Barc) 2001;117(10):377-385

9 Hosmer DWLS. Applied Logistic Regression. 2nd ed. Cressie NAC, editor. John Wiley \& Sons; 2000

10 Fong $\mathrm{K}$, Truong V, Foote $\mathrm{CJ}$, et al. Predictors of nonunion and reoperation in patients with fractures of the tibia: an observational study. BMC Musculoskelet Disord 2013;14:103

11 Gaebler C, Berger U, Schandelmaier P, et al. Rates and odds ratios for complications in closed and open tibial fractures treated with unreamed, small diameter tibial nails: a multicenter analysis of 467 cases. J Orthop Trauma 2001;15(06):415-423

12 Tzioupis C, Giannoudis PV. Prevalence of long-bone non-unions. Inj Int J Care Inj 2007:44

13 French B, Tornetta P III. High-energy tibial shaft fractures. Orthop Clin North Am 2002;33(01):211-230, ix

14 Metsemakers WJ, Handojo K, Reynders P, Sermon A, Vanderschot $\mathrm{P}, \mathrm{Nijs} \mathrm{S}$. Individual risk factors for deep infection and compromised fracture healing after intramedullary nailing of tibial shaft fractures: a single centre experience of 480 patients. Injury 2015; 46(04):740-745. Doi: 10.1016/j.injury.2014.12.018 [Internet] 